\title{
DISTRIBUTED SYSTEM OF REMOTE CONTROL AND MANAGEMENT OF TECHNOLOGICAL PROCESSES OF GRANARY
}

\author{
Anatoliy Bazhenov, Natalia Grivennaya, Sergey Malygin, Alexey Knyaginin \\ Don State Technical University, Russia \\ a_bazenov61@rambler.ru, katrinastenton@yandex.ru, malygin.sergei1959@yandex.ru, \\ a.knyaginin@gmail.com
}

\begin{abstract}
Technologies for storing agricultural products include heating, cooling and humidifying in order to protect the products from overcooling, overheating and dehydration. Due to improper storage conditions, farms suffer huge losses. When storing grain, it is necessary to know its temperature and humidity and conduct welltimed technological operations that ensure the grain's high-quality safety. The developed remote monitoring and control distributed system for the granary technological processes allows monitoring the temperature, relative humidity and concentration of ammonia both in the grain mass and headspace and controlling the cooling and heating processes, as well as maintaining the necessary humidity and air sanitation in the granary.
\end{abstract}

Keywords: agriculture, distributed system, monitoring, control, granary.

\section{Introduction}

The considerable attention of researchers all over the world is paid to the monitoring automation problems [1-3].

Currently, for measuring the temperature of grain stored in low-height grain bulks, portable rods and thermal probes are mainly used. Such measurements are often carried out in a manual way. According to recommendations, dry grain mass should be checked every 5-15 days, wet or damp grain must be checked daily. When the grain bulk is high (elevator silos, floored warehouses), special systems are used. The systems are based on remote measurement of grain temperature by an electric method. As a rule, the systems consist of temperature sensors, secondary devices, control circuits and communication elements. As sensors, low-resistance thermometers are in use. Logometers or balanced bridges are being used as secondary instruments $[4 ; 5]$.

The simplest is a system for remote measurement of grain temperature using a portable measuring device. If a secondary device is at the distance from the temperature measuring point, the temperature can be measured by means of auxiliary relay equipment that provides a choice of the required sensor and its connection to the secondary device. A measurement system is called remote, if the operator reads the measurement data from the secondary device. If the measurement procedure and data output of the measured parameter (temperature) occur automatically, the system is called remote-automatic.

Automation of the granary makes it possible to significantly reduce losses while storing grain. Most granary automated devices monitor only the temperature and/or humidity inside the grain mass and in the headspace. The moisture values in the grain mass and in the headspace differ significantly (7-16\% and 60-75\%, respectively). As a rule, monitoring of ammonia concentration as an indicator of the beginning of the grain mass rotting process is not carried out [5-8].

The system under development will allow real-time monitoring of the temperature and humidity of the grain mass, temperature, humidity, and ammonia concentration in the granary headspace. Threedimensional distribution of sensors in the grain mass will help identify damage sites at an early stage of their formation [9].

\section{Materials and methods}

Depending on the tasks to be solved, the structure of the automated system for controlling the technological processes in the granary may be of different types. If several granaries are under control, centralized management of them should be organized. To solve the task of controlling technological processes in large areas of granaries, it is advisable to use a system built on a distributed principle (DCS). Distributed Control System (DCS) is a technological process control system distinguishing by the design of a distributed input-output system and decentralized data processing. The DCS-system's main feature is a deep integration of code development tools for the visualization- and control levels [10].

Fig. 1 presents the structure of the distributed system for remote monitoring and control. 


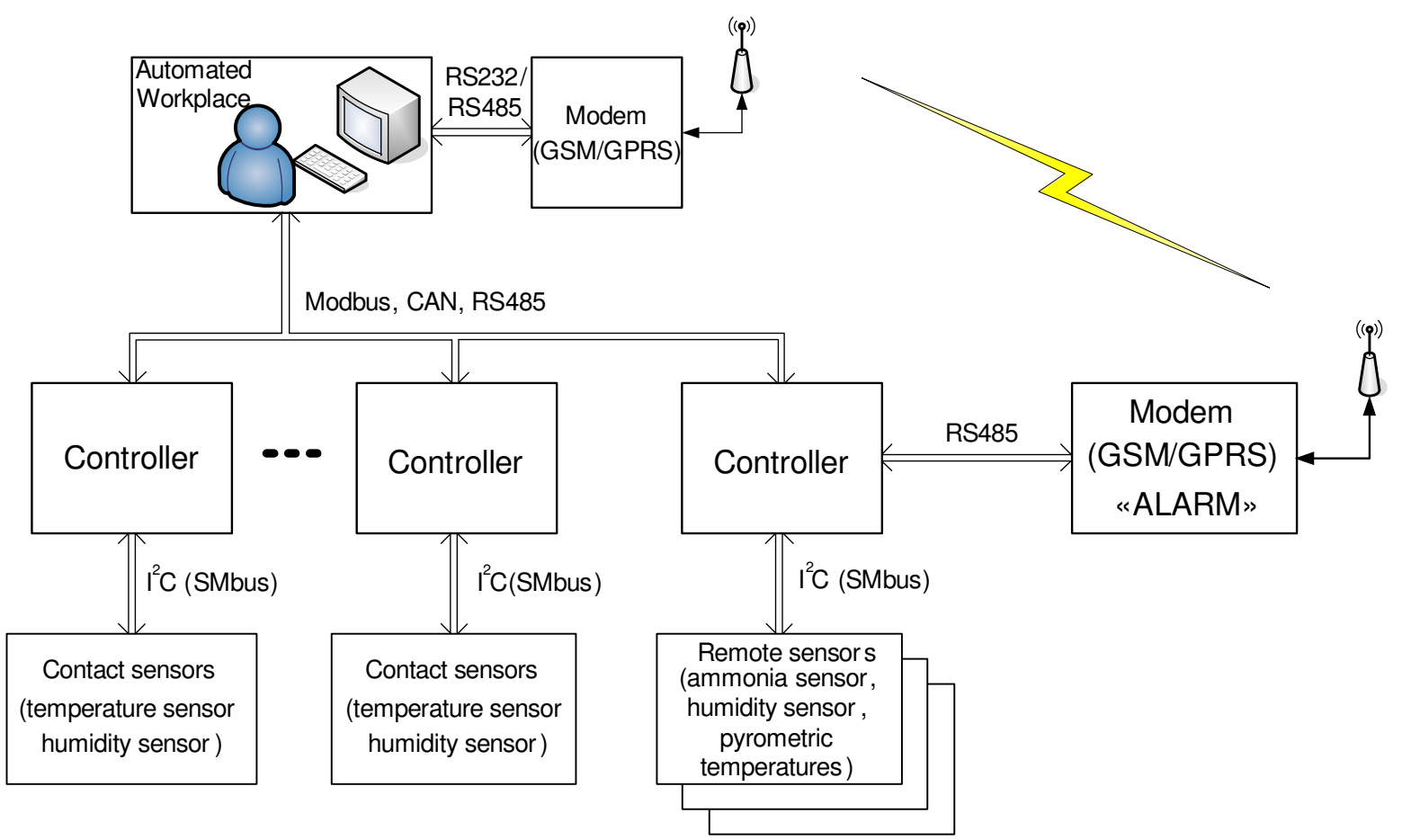

Fig. 1. Block diagram of developed remote monitoring and control distributed system

The system has a three-level structure. The lower I/O level contains sensors and actuators. The middle level consists of controllers that process the data received, form controlling actions and transfer data to the upper level. At the top level, there are database servers and operator stations (automated workstations); their task is to provide the operator human-machine interface and to exchange with the server and controllers. Controllers, input and output modules, sensors, actuators are spaced and interconnected by wired and wireless communication channels [11].

At the lower level, the control devices take the information off the sensors. The system allows connecting the following sensors:

- contact temperature sensors DS18S20 (Dallas Semiconductor) with programmable resolution from 9 to 12 bits, having a unique 64-bit serial code with exchange via a digital bus;

- non-contact temperature sensors (infrared thermometers) MXL90614 with SMBus output b;

- humidity sensors SHT75 (Sensirion), equipped with a 14-bit ADC and a serial interface circuit $\mathrm{I} 2 \mathrm{C}$;

- ammonia sensors DA-14-1 with digital output.

Distribution of functions of the system to several levels with partial duplication of information of sensors provides high fault tolerance of the system in general.

Digital temperature sensors make it possible to avoid many of the problems associated with the transmission of an analog signal from the sensor to the controller's input. These problems are due to the fact that the output of the thermal sensor is usually low-power and the transmission line of the analog signal is highly susceptible to electromagnetic fields and interference, which can significantly distort the measurement results. In addition, in case if the sensor is remote, the voltage drop on the line must also be taken into account, as it complicates the measurement results processing. When using a digital temperature sensor, the analog-to-digital signal conversion is performed directly on the chip. The data come to the receiver in a digitized form $[10 ; 12 ; 13]$.

Measurements of the grain mass temperature and the rates of temperature changes at different levels in the granaries are carried out using DS18S20 sensors, the temperature range of which is from minus $55^{\circ} \mathrm{C}$ to plus $125^{\circ} \mathrm{C}$ with an error of not more than $0.5^{\circ} \mathrm{C}$. The interrogation time of one sensor is $0.75 \mathrm{~s}$.

Measurement of the temperature in the headspace is carried out by means of infrared MXL90614 thermometers with a close to I2C SMBus output. The range of temperature determination on the 
remote surface is from minus $70{ }^{\circ} \mathrm{C}$ to plus $380^{\circ} \mathrm{C}$ with an error of not more than $0.5^{\circ} \mathrm{C}$. The result of the measurement is the averaged temperature of all the objects getting the working area of the sensor.

The relative humidity sensor SHT75 is a capacitive polymer humidity sensor that operates with a 14-bit analog-to-digital converter, a serial two-wire interface and an integrated voltage regulation system located in a single chip. Humidity measuring range is $0-100 \% \mathrm{rH}$ with an error of $2.0 \%$. The sensor response time is $4 \mathrm{sec}[14]$.

The ammonia sensor DA-14-2 with digital output is designed for automatic and continuous monitoring of ammonia concentration in the air. Response time for ammonia is $180 \mathrm{sec}$, the length of the communication line between the sensor and the control unit is up to $600 \mathrm{~m}$.

Thresholds for ammonia:

- warning $-200 \mathrm{mg} \cdot \mathrm{m}^{-3}$;

- alarm $-500 \mathrm{mg} \cdot \mathrm{m}^{-3}$.

The placement of sensors inside the granary is presented in Fig. 2. The number of sensors and servicing controllers is selected individually depending on the configuration of the object.

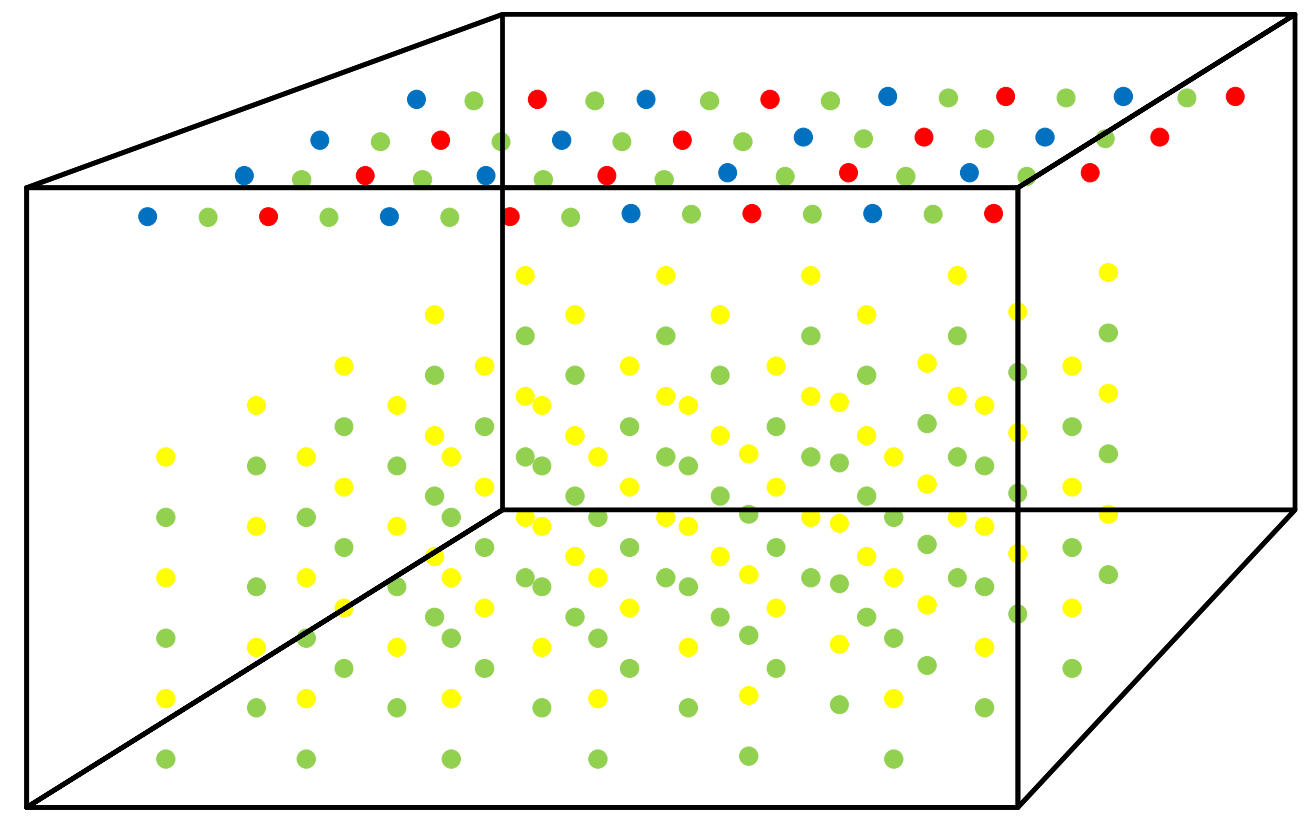

- ammonia sensor (DA-14-2)

- non-contact pyrometric temperature sensor(MLX90614)

contact temperature sensor (DS18B20)

- humidity sensor (SHT 75)

Fig. 2. Placement of sensors inside granary

All sensors in the multidrop mode have their own unique address; therefore, the access to each sensor goes to the corresponding address. The controller, which "serves" a group of sensors, detects all the sensors connected to the line, and can work with any of them.

The information received from the sensors enters the controllers, where it is processed. The connection scheme of the sensor cables to the dispatcher's workstation is shown in Fig.3. During the processing digital signals are converted into real values of temperature, humidity and ammonia concentration. This information is then transmitted to a personal computer and the dispatcher's workplace, where it is displayed in a real-time mode. The computer is continuously connected to the database server, where the results are stored. The information can be stored indefinitely, which allows getting records for any period of time [10].

Since all sensors are equipped with a digital interface, there is no need for additional equipment for interface conversion when connecting to mid-level controllers. 


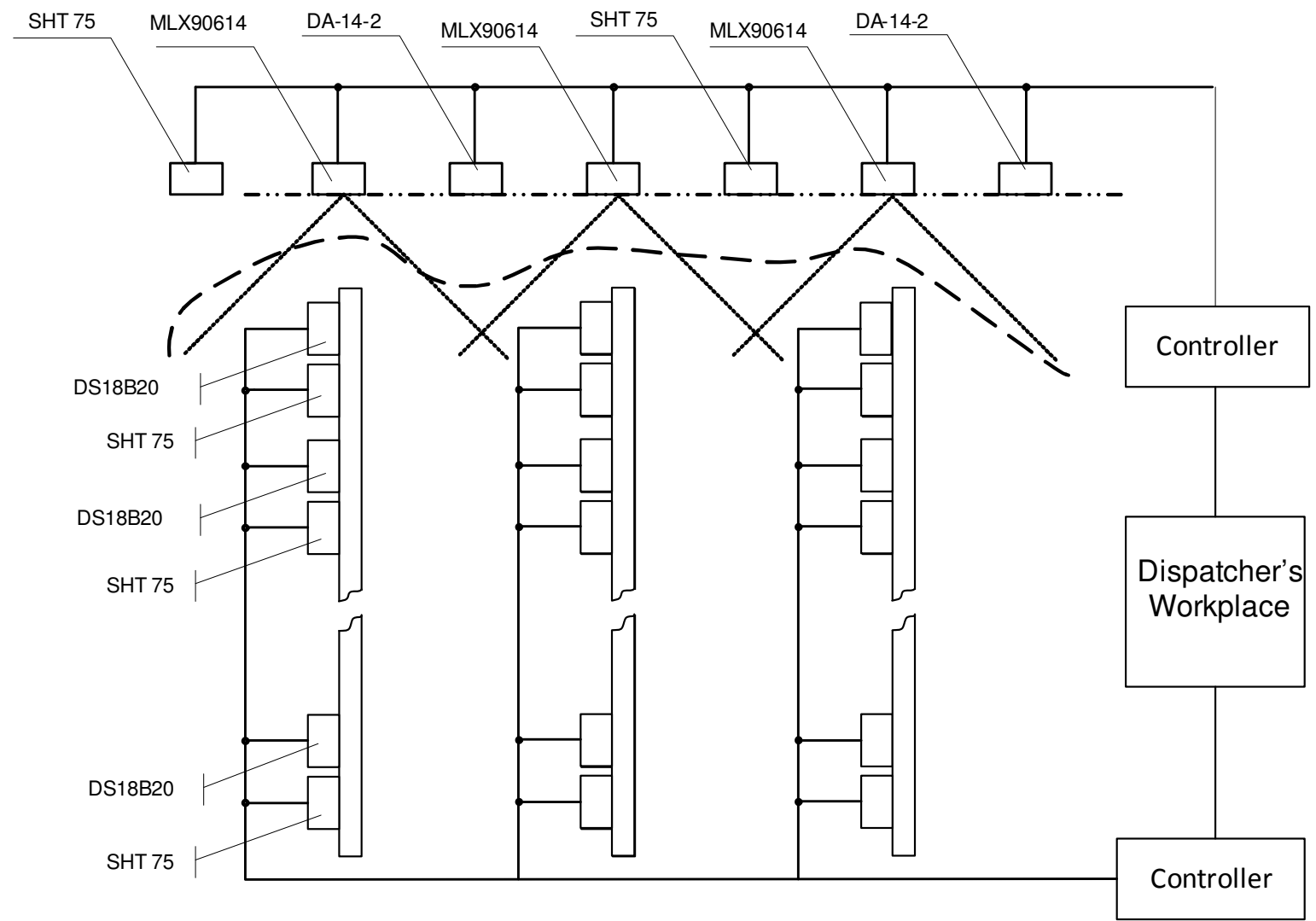

Fig. 3. Scheme connecting sensor cables to dispatcher's workstation

The middle level of the remote monitoring and control distributed system is formed of controllers that, under the control of the server, perform:

- receiving and transmitting data from sensors having different transmission rates and exchange protocols;

- converting the collected information into a single format;

- communication with the server of the distributed monitoring system via serial communication lines RS-485 under the CAN, Modbus, etc., protocols.

The maximum number of devices connected to one sensor controller via the I2C interface is limited by the load capacity of this interface and in the simplest case it is 128 .

The emergency controller is equipped with a GSM/GPRS modem and provides text messages about emergency situations via the GSM/GPRS channel to the server and the dispatcher's workstation. The emergency controller is connected to the sensor controllers via a digital interface RS-485 [11].

As the controllers that make up the system middle level, it is advisable to use the Arduino family boards, namely Arduino Uno, built on the ATmega328 microcontroller. The ATmega 328 controller possesses Flash, SRAM and EEPROM memory:

- FLASH - 32kB, of which $0.5 \mathrm{kB}$ is used to store the loader;

- $\quad$ SRAM (RAM) - 2kB;

- EEPROM - $1 \mathrm{kB}$ (it is available using the EEPROM library).

Each board has 6 analog inputs, 14 digital outputs of general purpose (can be both inputs and outputs), a $16 \mathrm{MHz}$ crystal oscillator, two sockets (power and USB), an ISCP connector for in-circuit programming and a hot reset button. For steady operation, the board must be powered either through the built-in USB connector or by connecting to the source from 7 to $12 \mathrm{~V}$. The board mainly differs from preceding boards of the Arduino Family in that it features the separate Atmega8U2 microcontroller instead of the FTDI programmer chip [15].

To communicate with external devices (a computer and other microcontrollers) there are several additional devices on the board. On the RX and TX pins, the ATmega328 controller supports an 
UART serial communication interface. ATmega8U2, which plays the programmer's role on the board, translates this interface via USB, allowing the platform to communicate with the computer via a standard COM port. The ATmega8U2 controller firmware uses standard USB-COM drivers, so no additional drivers are required for the connection.

The board provides communication via UART interface both by hardware and software. The board also supports the main interfaces for interaction with peripherals: SPI and I2C (TWI).

The upper level - the server system - consists of one or more servers working together and providing centralized monitoring and control of the granary technological processes.

The servers' software performs the following tasks:

- collects, processes, and stores information from sensors of physical values;

- makes a decision on the control signals generating for the object equipment;

- forms the user's graphical interface;

- controls the users' access to the system and their privileges in the system;

- combines several servers into a single information system and ensures the joint action of these servers.

The configuration of each server of the system is determined by the amount of object equipment connected to it. If the system is connected to the objects with the use of GSM- and Ethernet-networks, the server must be equipped with a transceiver GSM-modem and/or a network card respectively, to receive and transmit object information [16].

The dispatcher workstation is the user's access point to the system. It is an ordinary workstation connected to the Customer corporate Ethernet network.

The system has a Web-oriented interface and is accessed through a regular browser (Internet Explorer, for example). Therefore, when connecting a new dispatch computer no additional software is required to access the system. The maximum number of dispatcher workstations is practically unlimited.

The graphical interface of the system provides an intuitive color coding system to inform on the parameters: the color green corresponds to the normal non-emergency mode of operation; the color red indicates accidents, and yellow means lost connection.

The built-in graphical display tools allow creating screen images (mnemonic diagrams) of any complexity, including infinitely embedded in each other. Editing the structure of the object (removal or addition of equipment), as well as changing the parameters of its connection is carried out by the user through the Web-interface. Availability of the built-in library of devices and their drivers allows quick changing the composition of the object, if necessary, with a minimum time- and financial costing [11].

\section{Conclusions}

1. The proposed distributed system for remote monitoring and control in the granaries makes it possible to monitor the temperature, relative humidity and concentration of ammonia both in grain mass and in headspace and to control the processes of cooling, heating, maintaining the necessary humidity and air sanitation in the granary.

2. The developed system features decentralized processing of primary data and availability of distributed input and output systems.

3. The advantages of the given distributed system are increased fault tolerance, scalability, simple configuring, standard and unified database structure.

4. The main ideas of the developed system are used by the agricultural enterprises of Stavropol Region.

\section{References}

[1] Batey I. Chapter 20 - Maintaining Grain Quality During Storage and Transport, In Woodhead Publishing Series in Food Science, Technology and Nutrition. Woodhead Publishing, 2017, pp. 571-590. 
[2] Yu H., Zhang F., Wu Y. Granary: A sharing oriented distributed storage system. Future Generation Computer Systems, vol. 38, 2014, pp. 47-60.

[3] Song J. Measurement and Control System Based on Wireless Senor Network for Granary. Physics Procedia, vol. 24, Part A, 2012, pp. 566-571.

[4] Zhang H., Li X., Wang Y., Yao J., Kang Y., Wang Y. Evaluation and analysis of internal flow field uniformity in grain stack based on multi zone model of porous media. Procedia Engineering, vol. 205, 2017, pp. 2164-2170.

[5] Scariot M.A., Radünz L.L., Dionello R.G., Toni G.R., Mossi A.J., Reichert F.W. Jr. Quality of wheat grains harvested with different moisture contents and stored in hermetic and conventional system. Journal of Stored Products Research, vol. 75, 2018, pp. 29-34.

[6] Rosentrater K.A. and Evers A.D. Chapter 5 - Storage, handling and preprocessing. In Woodhead Publishing Series in Food Science, Technology and Nutrition. Woodhead Publishing, 2018, pp. 369-420.

[7] Liu X., Li B., Shen D., Cao J., Mao B. Analysis of Grain Storage Loss Based on Decision Tree Algorithm. Procedia Computer Science, vol. 122, 2017, pp. 130-137.

[8] Mohapatra D., Kumar S., Kotwaliwale N., Singh K.K. Critical factors responsible for fungi growth in stored food grains and non-Chemical approaches for their control. Industrial Crops and Products, vol. 108, 2017, pp. 162-182.

[9] Asefi M., Gilmore C., Jeffrey I., LoVetri J., Paliwal J. Detection and continuous monitoring of localised high-moisture regions in a full-scale grain storage bin using electromagnetic imaging. Biosystems Engineering, vol. 163, 2017, pp. 37-49.

[10]Dogan I. Chapter 6 - Integrated Circuit Temperature Sensors, In Microcontroller Based Temperature Monitoring and Control. Newnes, Oxford, 2002, pp. 129-143.

[11] Stangl M., Pielmeier J., Berger C., Braunreuther S., Reinhart G. Development of a Web Based Monitoring System for a Distributed and Modern Production. Procedia CIRP, vol. 52, 2016, pp. 222-227.

[12] Yurish S.Y. Digital sensors design based on universal frequency sensors interfacing IC, Sensors and Actuators A: Physical, vol. 132, Issue 1, 2006, pp. 265-270.

[13] Nawrocki W., Jurkowski A. and Pająkowski J. Chapter 220 - Digital Temperature Sensors in the Range of 77-300 K. In Proceedings of the Twentieth International Cryogenic Engineering Conference (ICEC20), Elsevier Science, Oxford, 2005, pp. 935-938.

[14] Aqeel-ur-Rehman, Abbasi A.Z., Islam N., Zubair A.S. A review of wireless sensors and networks' applications in agriculture. Computer Standards \& Interfaces, vol. 36, Issue 2, 2014, pp. 263-270.

[15]Pearce J.M. Chapter 4 - Open-Source Microcontrollers for Science: How to Use, Design Automated Equipment With and Troubleshoot. In Open-Source Lab, Elsevier, Boston, 2014, pp. 59-93.

[16] Pearce J.M., Chapter 6 - Digital Designs and Scientific Hardware. In Open-Source Lab, Elsevier, Boston, 2014, pp. 165-252. 